\title{
Seismic Moment-Rate and Longtoushan Records to Determinate Seismogenic Fault Direction of Ludian Ms6.5 Earthquake
}

\author{
Xueliang Chen ${ }^{1}$, Mengtan Gao ${ }^{1}$, Shicheng $\mathrm{Li}^{2}$, Jianwen $\mathrm{Cui}^{2}$, Jinping Guo ${ }^{1}$, Sen Qiao ${ }^{1}$ \\ ${ }^{1}$ Division of Engineering Seismology and Urban Disaster Mitigation; Key Laboratory of Seismic \\ Observation and Geophysical Imaging, Institute of Geophysics, China Earthquake Administration, \\ Beijing 100081, China \\ ${ }^{2}$ Disaster Prevention Research Institute, Yunnan Province Earthquake Administration, Kunming
}

650224, China

\section{地震矩率函数和龙头山记录研判鲁甸 6.5 级地震 发震断层方向}

\author{
陈学良 ${ }^{1}$, 高孟潭 ${ }^{1}$, 李世成 ${ }^{2}$, 崔建文 ${ }^{2}$, 郭金萍 ${ }^{1}$, 乔森 ${ }^{1}$ \\ ${ }^{1}$ 中国地震局地球物理研究所工程地震学与城市减灾研究室, 地震观测与地球物理成像重点实验 \\ 室, 北京 100081, 中国 \\ 2 云南省地震局防灾研究所, 昆明 650224 , 中国
}

\begin{abstract}
Three-component displacement time histories on the surface of near-field fault are obtained from analytical calculation of seismic moment release rate function and energy center source model. Analytical strong motion are compared with the strong motion records in meizoseismal area, the results indicated that, during Ludian Ms6.5 earthquake rupture process, NW-SE fault is the main seismogenic fault even if conjugated NE-SW direction fault also play the role in. This proposed method of using seismic moment release rate functions and strong motion records to determine comprehensively the dominant seismic fault direction, also can be used for other seismic event.
\end{abstract}

Keywords: seismic moment release rate function, strong motion records, comprehensive judgments, seismogenic fault.

\section{摘要}

通过能量中心点源模型和地震矩率函数的解 析计算, 得到了断层地表三分量位移时程, 与 极震区强震记录比较分析, 认为: 鲁甸 Ms6.5 地震破裂过程中, NW-SE (西北-东南) 向断 层为主要的发震断层, 即使与其共轭的 NE-SW 方向断层也参与的情况下。本文提出 的用地震矩率函数和强震记录综合研判发震 断层优势方向的方法, 亦可用于其他地震事件 研究。

关键词: 地震矩率函数, 强震记录, 综合研 判, 发震断层

1.引言

北京时间 2014 年 8 月 3 日 16 时 30 分 10 秒, 云南省昭通市鲁甸县 (北纬 $27.1^{\circ}$, 东经 $103.3^{\circ}$, 震源深度 $12 \mathrm{~km}$ ) 发生了 Ms6.5 地震 (简称鲁甸 6.5 级地震), 其烈度分布如图 1 所示 ${ }^{[16]}$ 。根据民政部报道, 截至 8 月 8 日 15 时, 本次地震已造成 617 人死亡, 112 人失踪, 


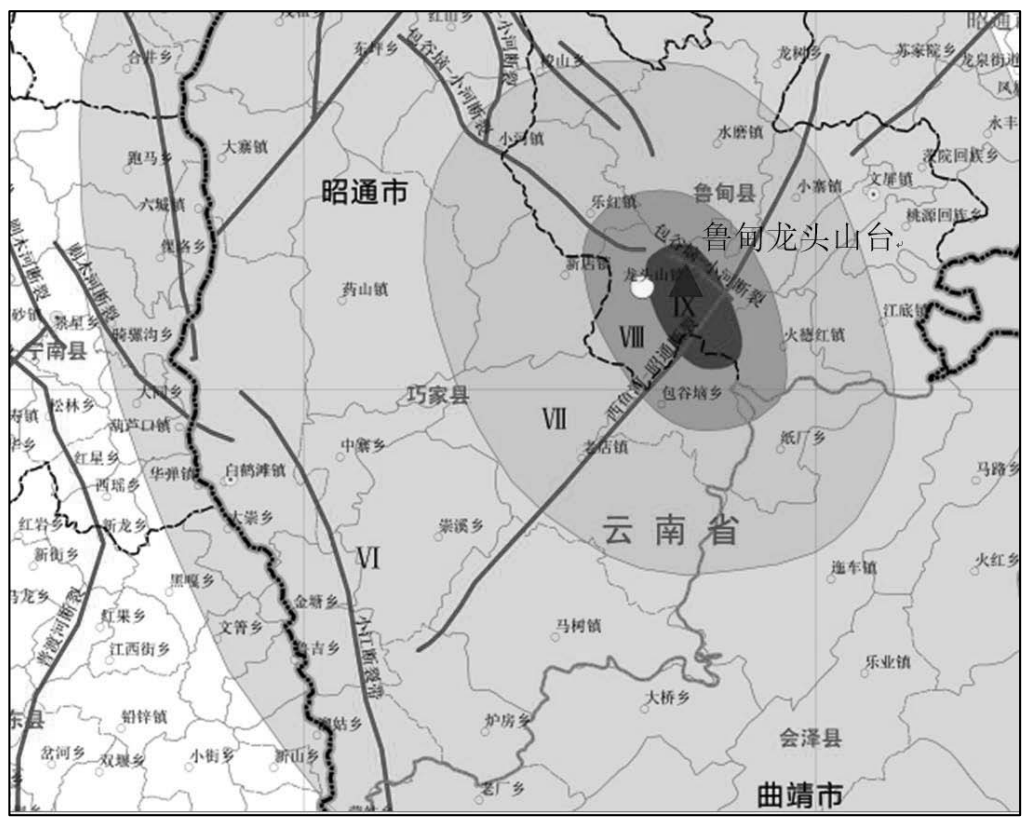

图 1 云南鲁甸 6.5 级地震烈度及龙头山强震台位置

3143 人受伤, 地震直接经济总损失 2357810 万元。总之，这是一次规模不大、但灾害尤为 严重的地震事件 ${ }^{[10-15]}$ 。

一些地质学者认为鲁甸 6.5 级地震的发震 断层为 NW 向包谷垴-小河断裂, 主要段落呈 左旋走滑性质(徐锡伟，陈桂华等，2013；徐 锡伟, 江国焰等, 2014; 赵翠萍, 周连庆等,

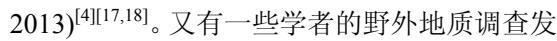
现, 龙头山镇政府所在地的大量地质滑坡和地 裂缝具有 NE 向优势分布特征, 且与 $\mathrm{NE}$ 向展 布的昭通-鲁甸断裂带关系密切（李西，张建 国等, 2014） ${ }^{[5]}$ 。震源破裂过程反演结果常倾 向于近南北向的小河-包谷垴断裂是此次地震 的发震构造 (张勇, 许力生等, 2014; 刘成利,

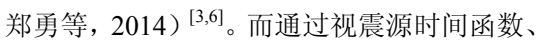
余震分布、进一步反演分析等结果表明, 鲁甸 地震可能是共轭断层先后破裂的结果 (许力生, 张旭等, 2014; 张广伟, 雷建设等, 2014; 张 勇, 陈运泰等, 2015） ${ }^{[7,8,9]}$ 。可以看出, 各位 学者对鲁甸 6.5 级地震发震断层的认识也不尽 一致。幸运的是, 极震区鲁甸龙头山强震台站 053LLT 获得了完整的强震动记录, 该记录中 必然蕴含了发震断层的震源机制和破裂过程 信息, 本文提出一种利用地震矩率函数和龙头
山强震记录相比较的简单方法, 尝试性地研判 了鲁甸 6.5 级地震的主要发震断层的优势方向。

\section{2. 极震区龙头山强震动台站附近建筑物震害}

位于极震区 (烈度IX度) 鲁甸龙头山镇财 政所大院内的龙头山 053LLT 强震台站, 其龙 头山强震动台站局部位置如图 2 所示, 龙头山 强震动台站仪器系统布置如图 3 所示。该台站 位于覆盖土层的自由地表, 所处场地属于 II 类 工程场地, 台站震中距为 $4.38 \mathrm{~km}$, 震源距为 $12.77 \mathrm{~km}$ 。

图 4 是 053LLT 台站的震害情况以及财政 所主楼、副楼、龙头山强震动台站和倒塌围墙

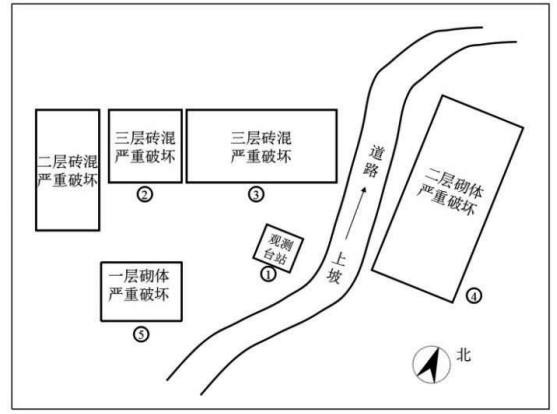

图 2 龙头山强震动台站局部位置示意图 
Risk Analysis and Crisis Response in Big Data Era (RAC-16)

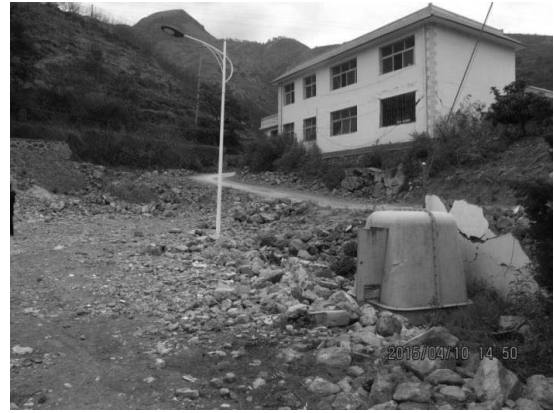

图 3 龙头山强震动台站台址, 该台东北方向的 二层砌体结构(4)震后已严重破坏

(倒塌方向为 NE 向)的位置关系。可以看出: 财政所办公楼主楼 (3)和副楼 (2)均为砖混结构, 地震前两者紧密联在一起, 地震后财政所办公 楼主楼(3)的底层 (一楼) 在地震动作用下“剪 毁”而“消失”，上部的第二层、第三层并未倒 塌。副楼(2)震后则严重破坏, 承重墙体出现较 严重的水平或“X”型贯通裂缝，但尚未倒塌。

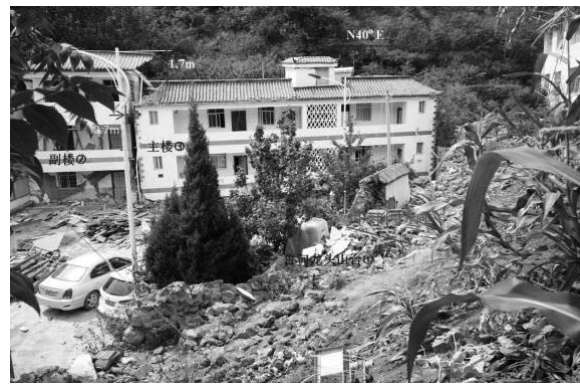

图 4 龙头山台的震害情况,财政所主楼、副楼、 台站及围墙（倒塌方向为 NE 向）的位置关系 主楼(3)楼体沿着楼体纵向 (长度方向) 即 $\mathrm{N} 40^{\circ} \mathrm{E}$ （北 $40^{\circ}$ 东方向）产生位移约 $70 \mathrm{~cm}$ ，导致主 楼 (3)和副楼(2)的高差约 $1.7 \mathrm{~m}$ 。该台站周围建筑 物震害的总体状况和震害指数均符合烈度 IX 度特征。从现场调查的房屋结构倒塌方向来看, NE-SW (东北-西南) 方向的倒塌数量确实比 NW-SE(西北-东南)方向倒塌数量要多一些, 大 部分房屋结构是 NE-SW(东北-西南)方向的倒 塌。

\section{053LLT 强震动记录}

龙头山台的三分量加速度时程在图 5 中
给出, 可以看出, 053LLT 的加速度记录的能 量集中区域的持续时间为 $20 \mathrm{~s}-35 \mathrm{~s}$, 总约 $15 \mathrm{~s}$, 记录到 EW 向强地面运动峰值加速度为 $948.5 \mathrm{gal}$, 另外两个方向, NS 向和 UD 向（坚 向）最大峰值加速度分别为-704.9gal 和 -503.9gal(崔建文, 刘琼仙等, 2014; 冀昆,

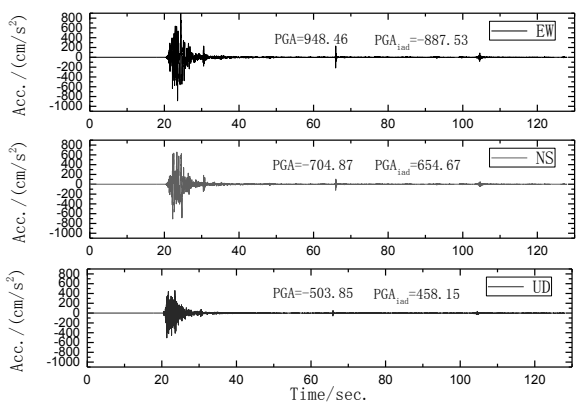

图 5 龙头山台的三分量加速度时程图

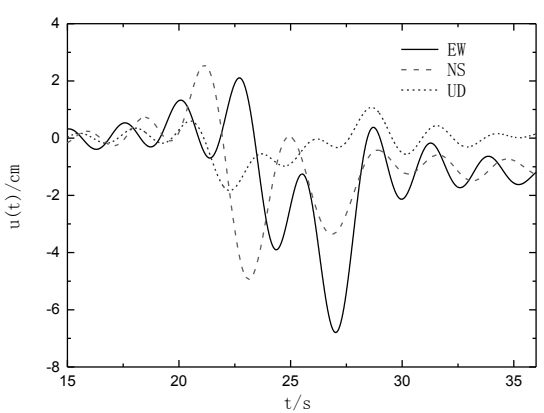

图 6 滤波后的龙头山位移时程的截取图

$(0.01-0.40 \mathrm{~Hz})$

温瑞智等, 2014 $)^{[12,13]}$ 。通过地震动常规处理 程序, 可以获得地震动位移时程。为了便于与 地震矩率函数得到的断层地表三分量位移时 程相比较, 将三分量位移时程带通滤波 $(0.01 \mathrm{~Hz}-0.40 \mathrm{~Hz})$, 并给出主要能量部分 $(15 \mathrm{~s}$ $-37 s$ ), 如图 6 所示, 位移时程经过滤波之后, 其 EW 向、NS 向和 UD 向位移峰值 PGD 分别 为 $-7 \mathrm{~cm} 、-5 \mathrm{~cm}$ 和 $-2 \mathrm{~cm}$, 可以看出, $\mathrm{EW}$ 向、 NS 向峰值位移 PGD 相对比较接近且两者的峰 值均较大, 而 UD 向幅值约为 EW 向和 NS 幅 值的 1/3-1/4。 
Risk Analysis and Crisis Response in Big Data Era (RAC-16)

\section{4 由地震矩率函数计算断层地表三分量位移时} 程 ${ }^{[1,2]}$

根据震源机制和破裂过程, 将地震破裂过程的 地震能量简化集中到能量中心处, 利用点剪切 位错源辐射理论和整体地震矩释放率函数, 可 以求得断层面能量中心投影到近场地表的 NS、 EW、UD 三分量理论位移时程。由于 053LLT 台站在震中附近, 理论位移时程与断层地表三 分量位移时程有相似关系。

根据文献[6], 取地球浅表第一层介质参数 $\mathrm{P}$ 波波速 $\alpha=5.1$ K n E , $\mathrm{S}$ 波波速 $\beta=2.8 \mathrm{Bm}$, 介质密度 $\rho=2.645 \mathrm{~g} / \mathrm{cm}^{3}$ 进行计算, 参考相关资料得到地表到能量中心 的距离 $R=7.071 \mathrm{~km}$ 。计算过程中用到的断 层模型几何参数示意图, 如图 7 所示, 根据文 献 $[1,2]$, 考虑近场、“中场”和远场项, 点剪切 位错源作用下, 近场地表的 NS、EW、UD 三 分量理论位移时程如式 (1) - (3) 所示。

\section{5 计算结果}

文献 [3] 利用了美国联邦地质调查局 (USGS) 体波震源机制解中的两个双力偶节 面: 节面 I: 走向 $72^{\circ}$ 、倾角 $84^{\circ}$ 、滑动角 $176^{\circ}$; 节面 II: 走向 $162^{\circ}$ 、倾角 $86^{\circ}$ 、滑动角 $6^{\circ}$ 。分 别选取两个节面作为发震断层面进行了震源 破裂过程反演 (张勇等, 2014) ${ }^{[3]}$, 结果如图 8、图 9 所示。

式(1)-(3)这三个时程公式中, 分别需要得 到 $\int_{R / \alpha}^{R / \beta} M_{0}(t-\tau) \tau d \tau 、 M_{0}\left(t-\frac{R}{\alpha}\right) 、 M_{0}\left(t-\frac{R}{\beta}\right)$ 、 $\mathrm{u}(\mathrm{r}, t)_{E W}=$

$\frac{\left[6 \sin \lambda \cos 2 \delta \cos \varphi_{s}-6 \cos \delta \cos \lambda \sin \varphi_{s}\right]}{4 \pi \rho R^{4}}$

$\times \int_{R / \alpha}^{R / \beta} M_{0}(t-\tau) \tau d \tau$

$+\frac{\left[2 \sin \lambda \cos 2 \delta \cos \varphi_{s}-2 \cos \delta \cos \lambda \sin \varphi_{s}\right]}{4 \pi \rho \alpha^{2} R^{2}}$

$\times M_{0}\left(t-\frac{R}{\alpha}\right)$

$+\frac{\left[3 \cos \delta \cos \lambda \sin \varphi_{s}-3 \sin \lambda \cos 2 \delta \cos \varphi_{s}\right]}{4 \pi \rho \beta^{2} R^{2}}$

$\times M_{0}\left(t-\frac{R}{\beta}\right)$

$+\frac{\left[\cos \delta \cos \lambda \sin \varphi_{s}-\sin \lambda \cos 2 \delta \cos \varphi_{s}\right]}{4 \pi \rho \beta^{3} R}$

$\times \dot{M}_{0}\left(t-\frac{R}{\beta}\right)$

$\mathrm{u}(\mathrm{r}, t)_{N S}=$

$\frac{\left[-6 \cos \delta \cos \lambda \cos \varphi_{s}-6 \sin \lambda \cos 2 \delta \sin \varphi_{s}\right]}{4 \pi \rho R^{4}}$

$\times \int_{R / \alpha}^{R / \beta} M_{0}(t-\tau) \tau d \tau$

$+\frac{\left[-2 \cos \delta \cos \lambda \cos \varphi_{s}-2 \sin \lambda \cos 2 \delta \sin \varphi_{s}\right]}{4 \pi \rho \alpha^{2} R^{2}}$

$\times M_{0}\left(t-\frac{R}{\alpha}\right)$

$+\frac{\left[3 \cos \delta \cos \lambda \cos \varphi_{s}+3 \sin \lambda \cos 2 \delta \sin \varphi_{s}\right]}{4 \pi \rho \beta^{2} R^{2}}$

$\times M_{0}\left(t-\frac{R}{\beta}\right)$

$+\frac{\left[\cos \delta \cos \lambda \cos \varphi_{s}+\sin \lambda \cos 2 \delta \sin \varphi_{s}\right]}{4 \pi \rho \beta^{3} R}$

$\times \dot{M}_{0}\left(t-\frac{R}{\beta}\right)$

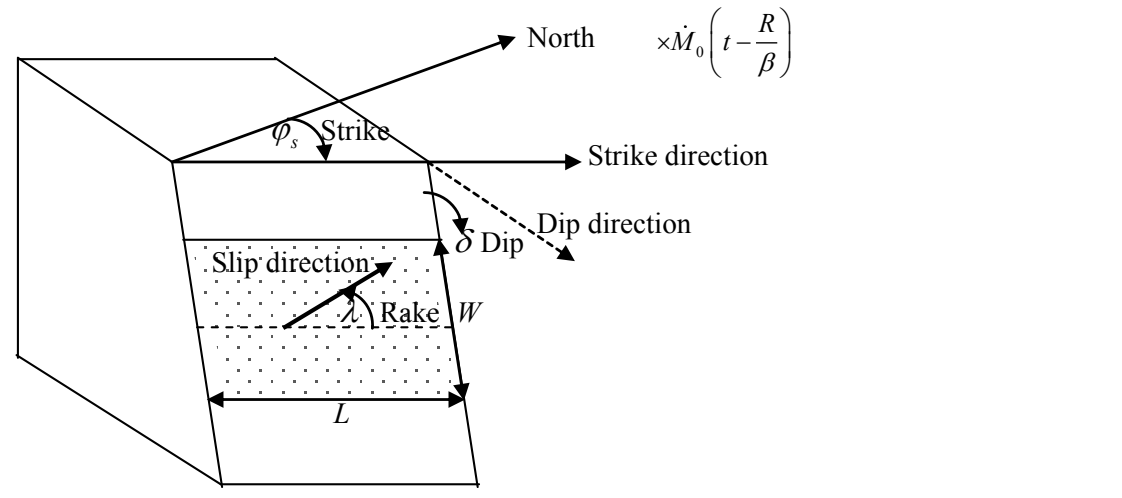

图 7 断层模型几何参数示意图 
Risk Analysis and Crisis Response in Big Data Era (RAC-16)

$$
\begin{aligned}
\mathrm{u}(\mathrm{r}, t)_{U D}= & \frac{[9 \sin \lambda \sin 2 \delta]}{4 \pi \rho R^{4}} \int_{R / \alpha}^{R / \beta} M_{0}(t-\tau) \tau d \tau \\
& +\frac{[4 \sin \lambda \sin 2 \delta]}{4 \pi \rho \alpha^{2} R^{2}} M_{0}\left(t-\frac{R}{\alpha}\right) \\
& +\frac{[-3 \sin \lambda \sin 2 \delta]}{4 \pi \rho \beta^{2} R^{2}} M_{0}\left(t-\frac{R}{\beta}\right) \\
& +\frac{[\sin \lambda \sin 2 \delta]}{4 \pi \rho \alpha^{3} R} \dot{M}_{0}\left(t-\frac{R}{\alpha}\right)
\end{aligned}
$$

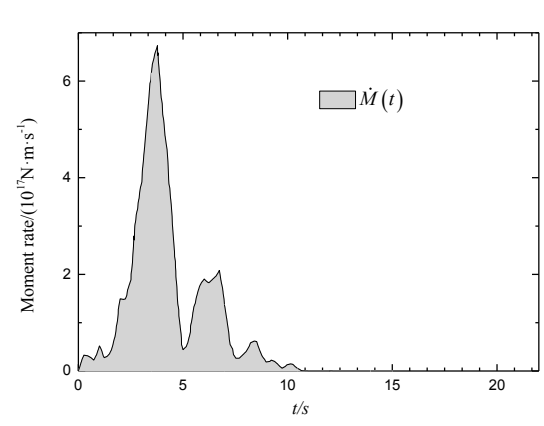

图 9 文献[3]北西向模型一（走向 $162^{\circ}$ 、倾角

$86^{\circ}$ 、滑动角 $\left.6^{\circ}\right)$ 的地震矩释放率函数

以及 $\dot{M}_{0}\left(t-\frac{R}{\alpha}\right)$ 和 $\dot{M}_{0}\left(t-\frac{R}{\beta}\right)$, 在此简要介绍 一下其含义: (1) $\dot{M}_{0}(t)$ 即为图 8, 图 9 所示 的地震矩释放率值, 时间间隔取为 $\Delta t=0.005 \mathrm{~s}$, 通过数学平移即可得到 $\dot{M}_{0}\left(t-\frac{R}{\alpha}\right)$ 和 $\dot{M}_{0}\left(t-\frac{R}{\beta}\right)$ 的时程。(2) 对(1)中所得到的两个 结果分别对时间积分, 可得到 $M_{0}\left(t-\frac{R}{\alpha}\right)$ 和 $M_{0}\left(t-\frac{R}{\beta}\right)$ 。但是, 这将导致时程结束时产生 很大的永久地震矩数值, 对于一个 Ms6.5 级的 中强地震, 可以认为地震结束后, 近断层地表 几乎不产生永久位移, 认为地震结束时该地震

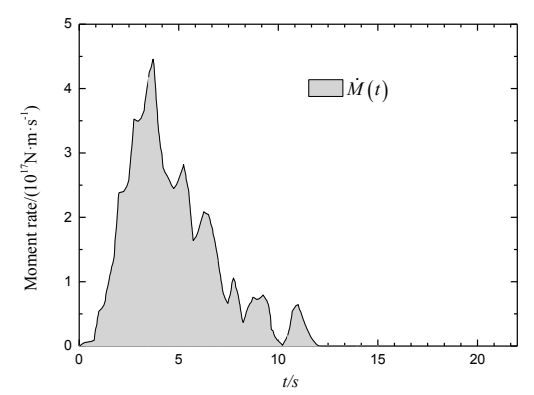

图 8 文献[3]北东向模型二(走向 $72^{\circ}$ 、倾角 $84^{\circ}$ 、

滑动角 $\left.176^{\circ}\right)$ 的地震矩释放率函数

矩为零。

本文采用经验性的抛物线轴消除漂移技 术, 取原曲线的第一个点和最后一个点为零点, 中间控制点取为中点 $\mathrm{Y}$ 轴的 0.25 倍, 三点组 成抛物线, 最后将积分曲线减去抛物线的, 得 到了消漂的 $M_{0}\left(t-\frac{R}{\alpha}\right)$ 和 $M_{0}\left(t-\frac{R}{\beta}\right)$ 。实践证 明, 这种方式取得了可观的效果。(3) 对于 $\int_{R / \alpha}^{R / \beta} M_{0}(t-\tau) \tau d \tau$ 的处理方法稍显复杂。由于 $M_{0}(t)$ 是一系列离散的点, 此积分可以表示成 $\sum_{R / \alpha}^{R / \beta} M_{0}(i \cdot \Delta t-j \cdot \Delta t) \cdot(j \cdot \Delta t) \cdot \Delta t$, 其 中 , $(j \cdot \Delta t) \in[R / \alpha, R / \beta] ; i \cdot \Delta t=t$ 。通过计算可 得到此项值。最将每幅图对应的走向, 倾角, 以及滑动角代入得到四个系数值, 依次代入三 个时程公式即可算出三个时程图, 如图 10(a)(b) 所示。 
Risk Analysis and Crisis Response in Big Data Era (RAC-16)

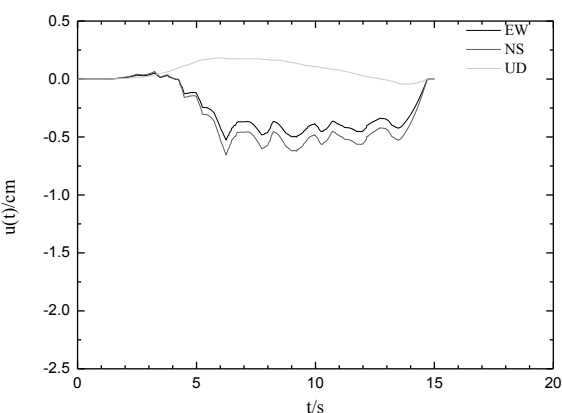

(a) 北东向模型二

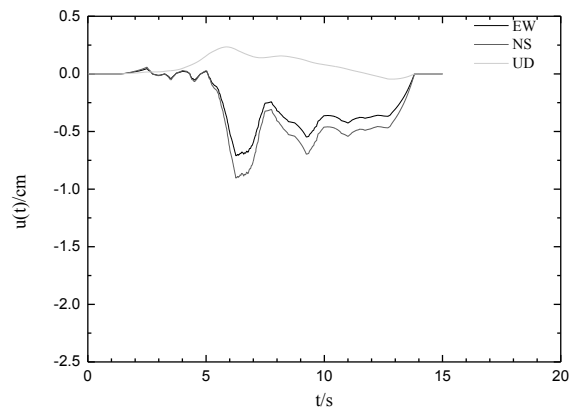

(b) 北西向模型一

图 10 两模型参数计算得到的 EW、NS 和 UD 向位移时程图

虽然上述结果（图 10）与实际地震波形 (图 6) 之间有较大差别, 但定性上看, 仍有 以下初步认识,

(1) 图 10(a)、(b)中坚向分量约为水平分量 的 1/3-1/4, 与图 6 中实际记录有相似之处。

(2) 与位移振动波形趋势的比较。图 10(b) 图的位移波形中明显有两个波谷一个波峰, 这 个振荡波形较贴近实际波形。图 10(a)位移波 形与实际相差都较大。

(3) 图 10(b)图表示西北-东南向的发震断 层。也就是说, 即使发震机制为共轭断层, 该 结果表明西北-东南向断层的断裂能量也将占 主要成分, 故本文认为鲁甸 Ms6.5 地震 NW-SE (西北-东南) 向断层为主要发震断层。
(4) 图 10(a)(b)中, NS 向的幅值比 EW 向 大, 这可能与鲁甸 Ms6.5 地震破裂过程的复杂 性有关系。

\section{6 结论与讨论}

通过对鲁甸 Ms6.5 地震震源破裂过程中 地震矩释放率函数的简单应用, 获得了近场地 表质点振动的三分量位移时程, 通过与实际地 震记录对比分析, 得到了理论上 EW 向位移反 应与 NS 向位移反应的量级基本相同, UD 向 位移反应较小, 约为水平分量的 1/3-1/4; 鲁甸 Ms6.5 地震破裂过程中, NW-SE (西北-东南) 向断层为主要的发震断层。

虽然理论计算得到的最大位移 PGD 约为 $1.0 \mathrm{~cm}-2.0 \mathrm{~cm}$ 量级, 而实际地震记录的 PGD 约 为 $5 \mathrm{~cm}-7 \mathrm{~cm}$, 这种差别或许是与波速较低浅层 地壳结构、浅表土层放大效应有关，053LLT 台站位于厚度为 26 米由杂填土、圆砾和砾砂 层构成的覆盖层上。

若条件允许, 建议采用复杂的震源断层运 动学或动力学模型, 开展相应的数值模拟分析, 以获得细致的理论地震动位移时程。

\section{致谢}

感谢中国地震局工程力学研究所负责的国家 强震动台网中心和云南省地震局提供的主震 观测数据。本文部分资料来自于云南鲁甸 6.5 级地震现场工作队。感谢中国地震局云南鲁甸 6.5 级地震现场队全体工作人员的辛勤工作。 本项研究受到 (喜马拉雅计划) 国家公益性行 业科研专题（201308011）、国家自然科学基金 面上项目（51278470）以及中国地震局地震观 测与地球物理成像重点实验室的资助。

\section{参考文献}

[1] Aki, K., Richards, P. G.(1980). Quantitative Seismology: Theory and Methods, vols.1 and 2. W. H. Freeman, San Francisco.

[2] 陈运泰, 震源理论基础, 北京: 中国地震局地 球物理研究所研究生教材, 2012.

[3] 张勇, 许力生, 陈运泰等. 2014. 2014 年 8 月 3 日云南鲁甸 $M_{\mathrm{w}} 6.1\left(M_{\mathrm{s}} 6.5\right)$ 地震破裂过程. 地球 物理学报, 57(9): 3052-3059.

[4] 徐锡伟, 江国焰, 等, 2014. 鲁甸 6.5 级地震发 
Risk Analysis and Crisis Response in Big Data Era (RAC-16)

震断层判定及其构造属性讨论，57（9）： 3060-3068

[5] 李西, 张建国, 等, 2014. 鲁甸 Ms6.5 地震地表 破坏及其构造的关系, 地震地质, 36（4): 1280-1292

[6] 刘成利, 郑勇, 熊熊等. 2014. 利用区域宽频带 数据反演鲁甸 $M_{\mathrm{s}} 6.5$ 级地震震源破裂过程. 地 球物理学报, 57(9): 3028-3037.

[7] 许力生, 张旭, 严川等. 2014. 基于勒夫波的鲁 甸 Ms6.5 地震震源复杂性分析 [J]. 地球物理学 报, 57(9): 3006-3017.

[8] 张广伟, 雷建设, 梁姗姗等. 2014. 2014 年 8 月 3 日云南鲁甸 Ms6.5 级地震序列重定位与震源 机制研究. 地球物理学报,57(9): 3018-3027.

[9] 张勇, 陈运泰, 等, 2014.2014 年云南鲁甸 $M_{W} 6.1$ 地震: 一次共轭破裂地震, 地球物理学报, 58 (1): 153-162.

[10] 中华人民共和国民政部网站，2014．http： //www.mca.gov.cn/article/zwgk/mzyw/201408/ 20140800678456.shtml

[11] 中国地震局网站, 2014 . http : //www.cea.gov.cn/publish/dizhenj/464/515/20140
80720182272 7235846/index.html

[12] 崔建文, 刘琼仙, 等, 2014. 2014 年云南鲁甸 6.5 级地震强震动观测记录及初步分析. 地震研 究, 37 (4): 542-548.

[13] 冀昆, 温瑞智, 崔建文, 等, 2014. 鲁甸 Ms6.5 级地震强震动记录及震害分析. 震灾防御技术, 9 (3): 325-339.

[14] 卢永坤, 张建国, 等, 2014. 2014 年云南鲁甸 6.5 级地震烈度分布于房屋震害特征. 地震研究, 37 (4): 549-557

[15] 非明伦, 2015. 云南地震灾害调查、烈度评定 及灾害损失评估工作 (2015.3), PPT.

[16] 乔森, 陈学良, 刘爱文, 等. 大陆首次超过 $1 \mathrm{~g}$ 的强地面运动记录特点及震害特征. 风险分析 和危机反应中的信息技术--中国灾害防御协会 风险分析专业委员会第六届年会论文集.2014.

[17]徐锡伟, 陈桂华, 于贵华, 等.芦山地震发震构 造及其与汶川地震关系讨论. 地学前缘, $20(3)$ : 11-20,2013.

[18] 赵翠萍, 周连庆, 陈章立.2013 年四川芦山 Ms7.0 级地震震源破裂过程及其构造意义. 科 学通报, 58(20): 1894-1900, 2013. 